\title{
Fixed points of $p$-toral groups acting on partition complexes
}

\author{
Julia E. Bergner, Ruth Joachimi, Kathryn Lesh, Vesna Stojanoska, \\ and Kirsten Wickelgren
}

\begin{abstract}
We consider the action of $p$-toral subgroups of $U(n)$ on the unitary partition complex $\mathcal{L}_{n}$. We show that if $H \subseteq U(n)$ is $p$-toral and has noncontractible fixed points on $\mathcal{L}_{n}$, then the image of $H$ in the projective unitary group $U(n) / S^{1}$ is an elementary abelian $p$-group.
\end{abstract}

\section{Introduction}

Let $\mathbf{n}$ denote the set $\{1, \ldots, n\}$ and let $\mathcal{P}_{n}$ denote the nerve of the poset of proper, nontrivial partitions of $\mathbf{n}$, ordered by coarsening. In ADL13, Arone, Dwyer, and Lesh compute the Bredon homology of $\mathcal{P}_{n}$ for certain kinds of $p$-local Mackey functors on the category of $\Sigma_{n}$-sets. The calculation is part of a program to obtain a proof of the Whitehead Conjecture and the collapse of the homotopy spectral sequence of the Goodwillie tower of the identity functor for $S^{1}$ by using the Bousfield-Kan cosimplicial resolution of $S^{1}$. A key element in the calculation of ADL13 is understanding which $p$-subgroups $H \subseteq \Sigma_{n}$ can have noncontractible fixed point sets $\left(\mathcal{P}_{n}\right)^{H}$. It turns out that if $H \subseteq \Sigma_{n}$ is a $p$-group and $\left(\mathcal{P}_{n}\right)^{H}$ is not contractible, then $H$ is elementary abelian (Proposition 6.6 in ADL13).

In this paper, we consider the corresponding question in the unitary context, following analogies set up by Arone and Lesh in Aro02 and [AL07. Let $\mathcal{L}_{n}$ denote the nerve of the (topological) poset of proper partitions of $\mathbb{C}^{n}$ into orthogonal subspaces, where "proper" means that we exclude the partition consisting of the single subspace $\mathbb{C}^{n}$ itself. (See Section 2.) The action of the unitary group $U(n)$ on $\mathbb{C}^{n}$ induces an action on $\mathcal{L}_{n}$. The space $\mathcal{L}_{n}$ with its $U(n)$ action is strongly related to the $b u$-analogues of symmetric powers of spheres and to the Weiss tower for the functor $V \mapsto B U(V)$ (see AL07 Theorem 9.5 and Aro02 Theorems 2 and 3).

In moving from finite group theory to compact Lie groups, one replaces the notion of a $p$-group with that of a $p$-toral group, i.e., an extension of a finite $p$ group by a torus. In this paper we study the action of $p$-toral subgroups $H \subseteq U(n)$ on $\mathcal{L}_{n}$ in order to find out when the fixed point set $\left(\mathcal{L}_{n}\right)^{H}$ is contractible. We show

2010 Mathematics Subject Classification. Primary 55N91, Secondary 55P65, 55R45.

The first, third, and fourth authors received partial support from NSF grants DMS-1105766, DMS-0968251, and DMS-1307390, respectively. The second author was partially supported by DFG grant HO 4729/1-1, and the fifth author was partially supported by an AIM 5-year fellowship. 
that the answer is "most of the time": the condition that $\left(\mathcal{L}_{n}\right)^{H}$ is not contractible puts considerable group-theoretic restrictions on the $p$-toral subgroup $H$.

Recall that the center of $U(n)$ is $S^{1}$, and that the projective unitary group is defined as $P U(n)=U(n) / S^{1}$. A subgroup $H$ of $U(n)$ is called projective elementary abelian if its image in $P U(n)$ is elementary abelian. Our main theorem is the following.

THEOREM 1.1. Suppose that $H$ is a p-toral subgroup of $U(n)$ and $\left(\mathcal{L}_{n}\right)^{H}$ is not contractible. Then $H$ is a projective elementary abelian p-subgroup of $U(n)$.

The organization of the paper is as follows. In Section 2, we define $\mathcal{L}_{n}$ and investigate the first nontrivial examples $(n=2,3)$ directly and in detail. Section 3 does some group-theoretic setup that is needed for studying fixed points. In Section 4 we find a condition on $H$ that implies contractibility of $\left(\mathcal{L}_{n}\right)^{H}$ (Theorem 4.5), and in Section 5 we prove Theorem 1.1 .

Finally, in Section 6 we compute two illustrative examples of fixed points. First, we exhibit a projective elementary abelian 2-subgroup of $U(2)$ whose fixed points on $\mathcal{L}_{2}$ are not contractible (Proposition 6.1), which shows that Theorem 1.1 is group-theoretically sharp. In contrast, we also show that the same subgroup, embedded in $U(3)$, has contractible fixed points on $\mathcal{L}_{3}$ (Proposition 6.3). In future work $\mathbf{B J L}^{+}$, we plan to establish further restrictions on the projective abelian $p$-subgroups of $U(n)$ that can have noncontractible fixed point sets by considering their representation theory in greater detail.

\section{Notation and Terminology}

We generally do not distinguish notation for a category and its nerve, and we trust to context to make clear which is being discussed.

By "subgroup," we always mean a closed subgroup. If $G$ is a group, we write $Z(G)$ for the center of $G$ and $G[p]$ for the elements of $G$ of order $p$.

Acknowledgements: The authors thank the Banff International Research Station and the Clay Mathematics Institute for financial support, and the anonymous referee for a helpful and thorough reading of the paper. The first and third authors are grateful to Bill Dwyer for preliminary discussions about this project.

\section{Homotopy type of $\mathcal{L}_{n}$ for small $n$}

In this section, we give an introduction to $\mathcal{L}_{n}$. We define and describe it in some detail, and we look at the two lowest-dimensional examples.

The partition complex $\mathcal{L}_{n}$ is a poset category internal to topological spaces. An object $\lambda$ in $\mathcal{L}_{n}$ is a proper, unordered decomposition of $\mathbb{C}^{n}$ into nonzero, mutually orthogonal subspaces $v_{1}, \ldots, v_{m}$, where by proper we mean that $m>1$. To topologize the set of objects, $\operatorname{Obj}\left(\mathcal{L}_{n}\right)$, let $\operatorname{Gr}_{k}\left(\mathbb{C}^{n}\right)$ denote the Grassmannian of $k$-planes in $\mathbb{C}^{n}$. The set of objects of $\mathcal{L}_{n}$ is given the subspace topology

$$
\operatorname{Obj}\left(\mathcal{L}_{n}\right) \subseteq \coprod_{m>1}\left[\left(\coprod_{k \geq 1} \operatorname{Gr}_{k}\left(\mathbb{C}^{n}\right)\right)^{m} / \Sigma_{m}\right]
$$

Note that the connected components $\operatorname{Obj}\left(\mathcal{L}_{n}\right)$ are in one-to-one correspondence with unordered partitions of the integer $n$ as the sum of at least two positive integers. 
Morphisms in $\mathcal{L}_{n}$ are given by coarsenings; that is, there is a morphism from $\left\{v_{1}, \ldots, v_{m}\right\}$ to $\left\{w_{1}, \ldots, w_{m^{\prime}}\right\}$ if and only if for each $v_{i}$ there exists a $j$ such that $v_{i} \subseteq w_{j}$. Note that between any two objects there is at most one morphism. In particular, there is a monomorphism

$$
\operatorname{Morph}\left(\mathcal{L}_{n}\right) \hookrightarrow \operatorname{Obj}\left(\mathcal{L}_{n}\right) \times \operatorname{Obj}\left(\mathcal{L}_{n}\right)
$$

via the source and target maps, and accordingly we topologize the morphism space by the subspace topology of the product topology.

Since $\mathcal{L}_{n}$ is a category internal to topological spaces, its nerve is a simplicial space (or the realization of that simplicial space, depending on context). Simplicial degree zero of the nerve consists simply of the space of objects of $\mathcal{L}_{n}$. The first simplicial degree consists of the space of morphisms, the second simplicial degree consists of the space of composable morphisms (topologized as a subspace of the two-fold product of the morphism space), and so forth. (See, for example, Section 5.1 of [Lib11.)

The action of $U(n)$ on $\mathbb{C}^{n}$ induces a continuous action of $U(n)$ on the category $\mathcal{L}_{n}$ (i.e., the action is continuous on the space of objects and the space of morphisms). Hence the nerve of $\mathcal{L}_{n}$ likewise has an action of $U(n)$, as do the nerves of any subcategories closed under the action of $U(n)$. By inspection of the simplices in the nerve of $\mathcal{L}_{n}$, we see that

$$
\text { (Nerve } \left.\left(\mathcal{L}_{n}\right)\right)^{H} \cong \operatorname{Nerve}\left(\left(\mathcal{L}_{n}\right)^{H}\right) \text {. }
$$

To provide the reader with some intuition about $\mathcal{L}_{n}$, at least in low dimensions, we work out concrete information about $\mathcal{L}_{2}$ and $\mathcal{L}_{3}$. (Observe that $\mathcal{L}_{1}$ is empty, since $\mathbb{C}$ has no proper partitions.) For $\mathcal{L}_{2}$, the smallest interesting example of a unitary partition complex, we can actually find the homeomorphism type. For the more complicated example of $\mathcal{L}_{3}$, we exhibit its homotopy type as a homotopy pushout diagram and prove that it is simply connected (Propositions 2.2 and 2.4).

To begin our study of $\mathcal{L}_{2}$, we observe that a proper partition of $\mathbb{C}^{2}$ can only be a partition into two orthogonal lines. Since there are no refinements and no proper coarsenings of such a partition, the poset category of partitions of $\mathbb{C}^{2}$ has only identity morphisms, and $\mathcal{L}_{2}$ is homeomorphic to its space of objects.

Proposition 2.1. The space $\mathcal{L}_{2}$ is homeomorphic to $\mathbb{R} P^{2}$.

Proof. A partition of $\mathbb{C}^{2}$ is an unordered pair consisting of a line in $\mathbb{C}^{2}$ and its orthogonal complement. The space of lines in $\mathbb{C}^{2}$ is the projective space $\mathbb{C} P^{1}$. Because the pair is unordered, $\mathcal{L}_{2}$ is the quotient of $\mathbb{C} P^{1}$ by the action of the involution that interchanges a line and its orthogonal complement.

More explicitly, note that the line spanned by $(0,1)$ has orthogonal complement spanned by $(1,0)$ (a special case), and in general the line in $\mathbb{C}^{2}$ spanned by $(1, z)$ with $z \in \mathbb{C} \backslash\{0\}$ has orthogonal complement spanned by $(1,-1 / \bar{z})$. Thus $\mathcal{L}_{2}$ is homeomorphic to the quotient of $S^{2} \cong \mathbb{C} P^{1} \cong \mathbb{C} \cup\{\infty\}$ by the involution $z \mapsto-1 / \bar{z}$ (and $0 \leftrightarrow \infty$ ). The involution exchanges points in the region $\|z\|>1$ with those in the region $\|z\|<1$, so we only need to consider the quotient of the unit disk $\|z\| \leq 1$ by the action on the boundary circle. When $\|z\|=1$, we can write $z=e^{i \varphi}$ and $-1 / \bar{z}=-e^{i \varphi}$ for some $\varphi \in \mathbb{R}$, whence the transformation is the antipodal map on the boundary of the unit disk. We conclude that $\mathcal{L}_{2}$ is homeomorphic to the quotient space obtained from the disk $\|z\| \leq 1$ by identifying antipodal points on the boundary circle, namely $\mathbb{R} P^{2}$. 
We now turn to $\mathcal{L}_{3}$, which is more complicated. There are two connected components in the space of objects, corresponding to partitions of $\mathbb{C}^{3}$ into three lines and partitions into a line and a 2-plane. The action of $U(n)$ on $\mathcal{L}_{n}$ allows the following explicit identification of the connected components of the object space: the transitive action of $U(n)$ on each connected component exhibits each component as the homogeneous space $U(n) / I$ once we have computed the isotropy group $I$ of a typical object in the component. A decomposition of $\mathbb{C}^{3}$ into a 1-dimensional subspace $v_{1}$ and its 2-dimensional orthogonal complement $v_{2}$ has isotropy group conjugate to $U(1) \times U(2)$, since an element of $U(3)$ that stabilizes the partition $\left\{v_{1}, v_{2}\right\}$ must stabilize $v_{1}$ and $v_{2}$ individually. On the other hand, elements of the isotropy group of a decomposition of $\mathbb{C}^{3}$ into three lines can act nontrivially on each line, but can also permute the lines, because the lines all have the same dimension. Hence this isotropy group is $(U(1))^{3} \rtimes \Sigma_{3}$. We conclude that the object space has homeomorphism type

$$
[U(3) /(U(1) \times U(2))] \sqcup\left[U(3) /\left((U(1))^{3} \rtimes \Sigma_{3}\right)\right] .
$$

We write $\operatorname{Gr}(1,2)$ for the first component, and $\operatorname{Gr}(1,1,1)$ for the second.

The next task is to identify the morphism space of $\mathcal{L}_{3}$. Each connected component of the object space gives a connected component of the morphism space consisting of identity morphisms, so two components of the morphism space of $\mathcal{L}_{3}$ are given by $\operatorname{Gr}(1,2)$ and $\operatorname{Gr}(1,1,1)$. (These components are precisely the degenerate simplices in simplicial dimension 1 of the nerve of $\mathcal{L}_{3}$.)

Unlike $\mathcal{L}_{2}$, the category $\mathcal{L}_{3}$ has nonidentity morphisms, given by coarsenings from $\operatorname{Gr}(1,1,1)$ to $\operatorname{Gr}(1,2)$. The action of $U(3)$ on these morphisms is transitive, so again we can identify the homeomorphism type of this component of the morphism space by finding the isotropy group of a sample morphism, say the morphism

$$
\mathbb{C} \oplus \mathbb{C} \oplus \mathbb{C} \longrightarrow \mathbb{C} \oplus \mathbb{C}^{2}
$$

that takes the standard basis to itself in the natural way. There is exactly one morphism between these objects, so for it to be fixed it is necessary and sufficient that both the source and the target be fixed. The isotropy group $I$ of the morphism is therefore the intersection of the isotropy groups of the source and the target, that is,

$$
\begin{aligned}
I & =\left(U(1)^{3} \rtimes \Sigma_{3}\right) \cap(U(1) \times U(2)) \\
& =U(1) \times\left(U(1)^{2} \rtimes \Sigma_{2}\right) .
\end{aligned}
$$

Proposition 2.2. The nerve of $\mathcal{L}_{3}$ is homeomorphic to the double mapping cylinder of the diagram

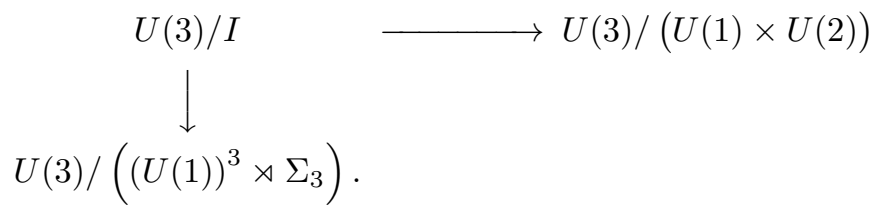

Proof. The nerve of the category $\mathcal{L}_{3}$ has nondegenerate simplices only in simplicial dimensions 0 and 1 , since there are no composable morphisms that do not involve an identity morphism. Diagram (2.3) has the two connected components of the object space of $\mathcal{L}_{3}$ in the upper right and lower left corners, and the nondegenerate part of the morphism space in the upper left corner. The double mapping 
cylinder is homeomorphic to the realization of the simplicial space that gives the nerve of $\mathcal{L}_{3}$.

From Proposition 2.2 we obtain the following homotopy-theoretic result.

Proposition 2.4. The space $\mathcal{L}_{3}$ is simply connected.

Proof. We use diagram (2.3) and the Seifert-Van Kampen theorem, noting that all of the spaces in (2.3) are path connected. To find the fundamental groups of the corners, recall that if $G$ is a compact Lie group with maximal torus $T$, then the natural map $\pi_{1} T \rightarrow \pi_{1} G$ is an epimorphism (e.g., Corollary 5.17 in [MT91). For the fundamental group of $U(3) /(U(1) \times U(2))$, we observe that $U(1) \times U(2)$ contains the maximal torus of $U(3)$ and so $U(1) \times U(2) \rightarrow U(3)$ induces a surjection on fundamental groups. Since $U(1) \times U(2)$ is connected, we conclude from the fiber sequence

$$
U(1) \times U(2) \rightarrow U(3) \rightarrow U(3) /(U(1) \times U(2))
$$

that the upper right corner of (2.3) is simply connected.

To find the fundamental group of the lower left corner of (2.3), we consider the fiber sequence

$$
U(1)^{3} \rtimes \Sigma_{3} \rightarrow U(3) \rightarrow U(3) /\left(U(1)^{3} \rtimes \Sigma_{3}\right) .
$$

Just as before, we find that the map $U(1)^{3} \rtimes \Sigma_{3} \rightarrow U(3)$ induces a surjection on fundamental groups. Continuing the long exact sequence in homotopy gives us

$$
\pi_{1}\left[U(3) /\left(U(1)^{3} \rtimes \Sigma_{3}\right)\right] \cong \pi_{0}\left[U(1)^{3} \rtimes \Sigma_{3}\right] \cong \Sigma_{3} .
$$

A similar argument tells us that in the upper left corner of (2.3), we have

$$
\pi_{1}[U(3) / I] \cong \pi_{0} I \cong \Sigma_{2},
$$

and the left vertical map on fundamental groups is the inclusion of $\Sigma_{2} \hookrightarrow \Sigma_{3}$ by $\left(\begin{array}{lll}1 & 2\end{array}\right) \mapsto(1)\left(\begin{array}{lll}2 & 3\end{array}\right)$.

The Seifert-Van Kampen theorem now tells us that the fundamental group of $\mathcal{L}_{3}$, which is the homotopy pushout of (2.3), is given by taking the free product of the fundamental groups of the lower left and upper right, namely $\Sigma_{3}$ and the trivial group, and taking the quotient by the normal subgroup generated by the fundamental group of the upper left corner, which is $\Sigma_{2}$. But the smallest normal subgroup of $\Sigma_{3}$ containing $\Sigma_{2}$ is actually $\Sigma_{3}$ itself. We conclude that $\pi_{1}\left(\mathcal{L}_{3}\right)$ is trivial.

\section{Group-theoretic results}

This section is devoted to establishing preliminary group-theoretic results about $U(n)$ and related groups that we will need in later sections. More precisely, the goal of this section is to establish criteria for $H \subseteq U(n)$ that allow us to find suitable elements of $H$ that are central and of order $p$. These criteria will be needed in order to apply Corollary 5.2 and construct contractions of fixed point spaces.

Let $P U(n)$ denote the projective unitary group, i.e., the quotient of $U(n)$ by its center $S^{1}$. If $H$ is a subgroup of $U(n)$, we use $\bar{H}$ to denote its image in $P U(n)$, so $\bar{H} \cong H /\left(S^{1} \cap H\right)$.

We recall the following terminology from Section 3.1 of GW98. Let $v$ be a complex representation of a group $G$; then $v$ splits into irreducibles as

$$
v \cong \bigoplus_{k} v_{k}^{\oplus m_{k}}
$$


where for different values of $k$, the corresponding irreducible representations $v_{k}$ are non-isomorphic. This decomposition is not canonical; however, if we group all the isomorphic irreducibles together into $w_{k}=v_{k}^{\oplus m_{k}}$, the decomposition

$$
v \cong \bigoplus_{k} w_{k}
$$

is canonical. It is called the isotypic decomposition of $v$, and we call the subspaces $w_{k}$ the isotypic components of $v$. If $v$ has only one isotypic component, we say it is an isotypic representation; otherwise, we say it is polytypic.

For our situation, we will usually be considering a closed subgroup $H \subseteq U(n)$ acting through the standard representation of $U(n)$ on $\mathbb{C}^{n}$. In this case we say that $H$ acts isotypically/polytypically or that $H$ is isotypic/polytypic if $\mathbb{C}^{n}$ is isotypic/polytypic as an $H$-representation.

Lemma 3.1. Let $\mathbb{Z} / p$ be a subgroup of $P U(n)$ and let $J$ be its inverse image in $U(n)$. Then $J$ is polytypic. In fact, a generator of $\mathbb{Z} / p \subseteq P U(n)$ can be lifted to an element of order $p$ in $U(n)$ and $J \cong S^{1} \times \mathbb{Z} / p$.

Proof. Let $A \in U(n)$ be such that its image $\bar{A} \in P U(n)$ generates the given $\mathbb{Z} / p$. Then $A^{p}$ is an element of the central $S^{1} \subseteq U(n)$ and is a diagonal matrix with all equal diagonal entries. Let $\alpha$ be some $p$ th root of the diagonal entry of $A^{p}$. Define $B=\alpha^{-1} A$, so $B^{p}=\mathrm{Id}$ and the image of $B$ in $P U(n)$ is $\bar{A}$. Then $\bar{A} \mapsto B$ determines a homomorphism that splits the short exact sequence

$$
1 \rightarrow S^{1} \rightarrow J \rightarrow \mathbb{Z} / p \rightarrow 1,
$$

and since $S^{1}$ is central, the map $S^{1} \times \mathbb{Z} / p \rightarrow J$ given by $\left(s, \bar{A}^{b}\right) \mapsto s B^{b}$ respects the multiplication in $J$ and is an isomorphism.

It remains to show that $J$ is polytypic. Since $J$ is abelian, its irreducible representations are all one-dimensional, so every element of $J$ acts on every onedimensional $J$-irreducible summand of $\mathbb{C}^{n}$ by multiplication by a scalar. If $J$ were isotypic, then an element of $J$ would have to act on every such summand by multiplication by the same scalar, i.e., $J$ would be contained in $S^{1}$, which is false. Hence, $J$ is polytypic.

The subgroups of $U(n)$ of greatest interest to us are the $p$-toral subgroups. We begin with a definition.

Definition 3.2. A p-toral group $H$ is an extension of a finite $p$-group by a torus. In other words, there exists a short exact sequence

$$
1 \rightarrow T \rightarrow H \rightarrow P \rightarrow 1,
$$

where $T$ is a torus and $P$ is a finite $p$-group. If $T \cong\left(S^{1}\right)^{\times r}$, we call $r$ the rank of $H$. The torus $T$ is the connected component of the identity of $H$, so we also denote it by $H_{0}$.

Lemma 3.3. Any quotient of a p-toral subgroup $H$ by a closed normal subgroup $K$ is p-toral.

In particular, if $H$ is a $p$-toral subgroup of $U(n)$, then its image $\bar{H}$ in $P U(n)$ is also $p$-toral. 
Proof. Since $H$ is $p$-toral, we have a short exact sequence

$$
1 \rightarrow T \rightarrow H \rightarrow P \rightarrow 1,
$$

where $T$ is a torus and $P$ is a $p$-group. We obtain a morphism of short exact sequences

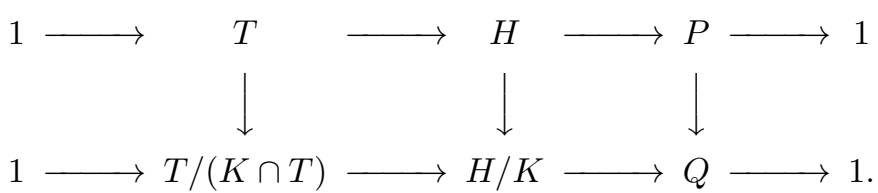

The map $H \rightarrow H / K$ is surjective, as is $H / K \rightarrow Q$, so $P \rightarrow Q$ is surjective, implying that $Q$ is a $p$-group. Further, $T /(K \cap T)$ is a compact connected abelian Lie group, and so must be a torus. in $\bar{H}$.

For the remainder of this section, we focus on finding certain elements of order $p$

Definition 3.4. For a $p$-toral group $H$, let $H / p$ denote the quotient of $H$ by its normal subgroup $H^{p}[H, H]$, the normal subgroup generated by $p$-th powers and commutators.

For a finite group $H$, the subgroup $H^{p}[H, H]$ may be familiar to the reader as the Frattini subgroup of $H$, and $H / p$ as the Frattini quotient of $\mathrm{H}$. A $p$-toral group $H$ is elementary abelian if and only if both $H^{p}$ and $[H, H]$ are the trivial subgroup of $H$, i.e., if and only if $H^{p}[H, H]$, the subgroup generated by both, is trivial. Further, $H / p$ is an elementary abelian $p$-group, and the map $H \rightarrow H / p$ is initial among homomorphisms from $H$ to finite elementary abelian $p$-groups; thus we refer to $H / p$ as the maximal elementary abelian quotient of $H$.

The following lemma is the main technical tool for the proof of Theorem 1.1 in Section 5] Part of the statement is actually Lemma 6.5 of [ADL13], for which we have given a streamlined proof here.

LEMMA 3.5. Let $H$ be a p-toral subgroup of $U(n)$ with image $\bar{H}$ in $P U(n)$. If $\bar{H}$ is nontrivial and not elementary abelian, then there exists a central element in $\bar{H}$ of order $p$ that lies in the kernel of $\bar{H} \rightarrow \bar{H} / p$.

Proof. By Lemma 3.3, $\bar{H}$ is itself $p$-toral. If $\bar{H}$ is connected, then it is a (nontrivial) torus and has at least $p-1$ elements of order $p$. They are central in $\bar{H}$ because the torus is abelian, and map to the identity in $\bar{H} / p$ because a torus is $p$-divisible.

Suppose that $\bar{H}$ is not connected and has a nontrivial identity component $\bar{H}_{0}$. Let $\bar{H}_{0}[p]$ denote the group of elements of $\bar{H}_{0}$ that have order $p$. The conjugation action of $\bar{H}$ on itself preserves $\bar{H}_{0}[p]$, while $\bar{H}_{0}$ acts trivially on $\bar{H}_{0}[p]$ since $\bar{H}_{0}$ is abelian, so we get an action of $\bar{H} / \bar{H}_{0}$ on $\bar{H}_{0}[p]$. By assumption $\bar{H}_{0}$ is nontrivial, so the set $\bar{H}_{0}[p]$ has $p^{\operatorname{rank}\left(\bar{H}_{0}\right)}>1$ elements. The nontrivial $p$-group $\bar{H} / \bar{H}_{0}$ fixes the identity element in $\bar{H}_{0}[p]$, so by the orbit decomposition of $\bar{H}_{0}[p]$, there exist at least $p-1$ other elements of $\bar{H}_{0}[p]$ fixed by $\bar{H} / \bar{H}_{0}$. These elements are in $Z(\bar{H})$, and because $\bar{H}_{0}$ is a torus and is $p$-divisible, we know they also map to the identity in $\bar{H} / p$.

Now suppose that $\bar{H}$ is not connected and has trivial identity component, i.e., $\bar{H}$ is a finite $p$-group. Since $\bar{H}$ is not elementary abelian, the subgroup $\bar{H}^{p}[\bar{H}, \bar{H}]$ is 
a nontrivial subgroup of $\bar{H}$, and it is also normal. But a nontrivial normal subgroup of a finite $p$-group must have nontrivial intersection with the center, and this gives the required element.

\section{Conditions for contractibility of the fixed point sets}

In this section, we turn to $\mathcal{L}_{n}$ for a general $n$ and establish preliminary criteria for the action of a subgroup $H \subseteq U(n)$ on $\mathcal{L}_{n}$ to have a contractible fixed point set (Proposition 4.2 and Theorem 4.5). We begin with some terminology and notation. We often think of an object $\lambda$ in $\mathcal{L}_{n}$ as given by the equivalence classes of an equivalence relation $\sim_{\lambda}$ on $\mathbb{C}^{n} \backslash\{0\}$, where $x \sim_{\lambda} y$ if $x$ and $y$ are in the same subspace of the partition $\lambda$. We therefore denote the set of subspaces of the partition $\lambda$ by $\operatorname{cl}(\lambda):=\left\{v_{1}, \ldots, v_{m}\right\}$.

DeFinition 4.1.

(1) A partition $\lambda$ is weakly fixed by $H$, or weakly $H$-fixed if $x \sim_{\lambda} y$ implies $h x \sim_{\lambda} h y$ for all $h \in H$. That is, the action of $H$ on $\mathbb{C}^{n}$ stabilizes $\operatorname{cl}(\lambda)$ as a set, although $H$ may permute the elements of $\operatorname{cl}(\lambda)$ nontrivially. We denote the full subcategory of $\mathcal{L}_{n}$ whose objects are weakly $H$-fixed partitions of $\mathbb{C}^{n}$ by $\left(\mathcal{L}_{n}\right)^{H}$.

(2) A partition $\lambda$ is strongly fixed by $H$, or strongly $H$-fixed, if $x \sim_{\lambda} h x$ for all $x \in \mathbb{C}^{n} \backslash\{0\}$ and all $h \in H$. That is, the action of $H$ on $\operatorname{cl}(\lambda)$ is trivial. We denote the full subcategory of $\mathcal{L}_{n}$ whose objects are strongly $H$-fixed partitions of $\mathbb{C}^{n}$ by $\left(\mathcal{L}_{n}\right)_{\mathrm{st}}^{H}$.

(3) A strongly $H$-fixed partition $\lambda$ is called $H$-isotypic if each element of $\operatorname{cl}(\lambda)$ is an isotypic representation of $H$. We denote the full subcategory of $H$-isotypic partitions by $\left(\mathcal{L}_{n}\right)_{\text {iso }}^{H}$.

We observe that

$$
\left(\mathcal{L}_{n}\right)_{\text {iso }}^{H} \subseteq\left(\mathcal{L}_{n}\right)_{\mathrm{st}}^{H} \subseteq\left(\mathcal{L}_{n}\right)^{H},
$$

and that in general the containments are strict. We are interested in conditions under which $\left(\mathcal{L}_{n}\right)^{H}$ is contractible.

Proposition 4.2. Let $H \subseteq U(n)$ be connected and polytypic. Then $\left(\mathcal{L}_{n}\right)^{H}$ is contractible.

Remark. Proposition 4.2 is not actually used directly in the proof of Theorem 1.1. We nonetheless include it as it may be of independent interest, and it provides an uncomplicated exemplar of our methods.

Proof. The action of $H$ on the elements of $\operatorname{cl}(\lambda)$ for $\lambda \in\left(\mathcal{L}_{n}\right)^{H}$ defines a continuous map

$$
H \rightarrow \Sigma_{\mathrm{cl}(\lambda)}
$$

from $H$ to the symmetric group on $\operatorname{cl}(\lambda)$. Since $H$ is connected and $\Sigma_{\mathrm{cl}(\lambda)}$ is discrete, this map is trivial. Thus any weakly $H$-fixed partition must be strongly $H$-fixed, and it is sufficient to prove that $\left(\mathcal{L}_{n}\right)_{\mathrm{st}}^{H}$ is contractible.

A strongly $H$-fixed partition is a decomposition of $\mathbb{C}^{n}$ into representations of $H$. Each of these representations can in turn be decomposed into its isotypic components as an $H$-representation, which defines a functor $\phi:\left(\mathcal{L}_{n}\right)_{\mathrm{st}}^{H} \rightarrow\left(\mathcal{L}_{n}\right)_{\text {iso }}^{H}$. There is also a natural transformation of the composite

$$
\left(\mathcal{L}_{n}\right)_{\mathrm{st}}^{H} \stackrel{\phi}{\longrightarrow}\left(\mathcal{L}_{n}\right)_{\mathrm{iso}}^{H} \hookrightarrow\left(\mathcal{L}_{n}\right)_{\mathrm{st}}^{H}
$$


to the identity functor on $\left(\mathcal{L}_{n}\right)_{\mathrm{st}}^{H}$, while the other composition is actually equal to the identity. It follows that $\left(\mathcal{L}_{n}\right)_{\mathrm{st}}^{H}$ is homotopy equivalent to $\left(\mathcal{L}_{n}\right)_{\text {iso }}^{H}$. However, $H$ is polytypic, so the decomposition of $\mathbb{C}^{n}$ into isotypic components is a proper partition of $\mathbb{C}^{n}$. This partition is a terminal object of $\left(\mathcal{L}_{n}\right)_{\text {iso }}^{H}$, whence $\left(\mathcal{L}_{n}\right)_{\text {iso }}^{H}$ is contractible.

Recall that any partition $\lambda$ in $\mathcal{L}_{n}$ corresponds to an equivalence relation on points of $\mathbb{C}^{n} \backslash\{0\}$, and $x \sim_{\lambda} y$ if $x$ and $y$ are in the same subspace of the partition $\lambda$. We now define another, coarser equivalence relation which incorporates the group action.

Definition 4.3. Let $J \subseteq U(n)$ be a subgroup, and let $\lambda$ be an element of $\mathcal{L}_{n}$ corresponding to the relation $\sim_{\lambda}$. We define a new equivalence relation $\sim_{(\lambda / J)}$ by $x \sim_{(\lambda / J)} y$ if there exists $j \in J$ such that $x \sim_{\lambda} j y$, and we denote the associated partition by $(\lambda / J)$.

In other words, the partition $(\lambda / J)$ is the minimal coarsening of $\lambda$ that is strongly fixed by $J$.

Lemma 4.4. Let $J$ be a normal subgroup of $H$ and let $\lambda \in\left(\mathcal{L}_{n}\right)^{H}$. Assume that $(\lambda / J)$ is a proper partition of $\mathbb{C}^{n}$. Then $(\lambda / J) \in\left(\mathcal{L}_{n}\right)^{H}$.

Proof. We want to show that $(\lambda / J)$ is weakly $H$-fixed. Let $h \in H$ and suppose that $x \sim_{(\lambda / J)} y$. By definition of $(\lambda / J)$, there exists some $j \in J$ such that $x \sim_{\lambda} j y$. Since $\lambda$ is fixed by $H$, we have $h x \sim_{\lambda} h j y=\left(h j h^{-1}\right) h y$. Since $J$ is normal in $H$, the element $h j h^{-1}$ is in $J$, so $h x \sim_{(\lambda / J)} h y$.

We now bring these results together to give conditions under which $\left(\mathcal{L}_{n}\right)^{H}$ is contractible.

TheOREM 4.5. Let $H \subseteq U(n)$, and let $J$ be a normal subgroup of $H$ such that for every $\lambda \in\left(\mathcal{L}_{n}\right)^{H}$, the partition $(\lambda / J)$ is a proper partition of $\mathbb{C}^{n}$. If $J$ is polytypic, then

$$
\left(\mathcal{L}_{n}\right)^{H} \simeq\left(\mathcal{L}_{n}\right)^{H} \cap\left(\mathcal{L}_{n}\right)_{\text {iso }}^{J}
$$

and $\left(\mathcal{L}_{n}\right)^{H}$ is contractible.

Proof. Under our assumptions and by Lemma 4.4, the assignment $\lambda \mapsto(\lambda / J)$ defines a functor

$$
\left(\mathcal{L}_{n}\right)^{H} \rightarrow\left(\mathcal{L}_{n}\right)^{H} \cap\left(\mathcal{L}_{n}\right)_{\text {st }}^{J} .
$$

Since $(\lambda / J)$ is a coarsening of $\lambda$, there is a natural transformation from the identity functor on $\left(\mathcal{L}_{n}\right)^{H}$ to the composite

$$
\left(\mathcal{L}_{n}\right)^{H} \rightarrow\left(\mathcal{L}_{n}\right)^{H} \cap\left(\mathcal{L}_{n}\right)_{\text {st }}^{J} \hookrightarrow\left(\mathcal{L}_{n}\right)^{H},
$$

showing that the induced composite map on classifying spaces is homotopic to the identity. Since for $\lambda$ in $\left(\mathcal{L}_{n}\right)^{H} \cap\left(\mathcal{L}_{n}\right)_{\text {st }}^{J}$, we know $(\lambda / J)=\lambda$, it follows that the map on classifying spaces induced by the functor $\lambda \mapsto(\lambda / J)$ is a deformation retraction, giving a homotopy equivalence

$$
\left(\mathcal{L}_{n}\right)^{H} \simeq\left(\mathcal{L}_{n}\right)^{H} \cap\left(\mathcal{L}_{n}\right)_{\mathrm{st}}^{J} .
$$

Next, we show that $\left(\mathcal{L}_{n}\right)^{H} \cap\left(\mathcal{L}_{n}\right)_{\text {iso }}^{J} \hookrightarrow\left(\mathcal{L}_{n}\right)^{H} \cap\left(\mathcal{L}_{n}\right)_{\text {st }}^{J}$ is a homotopy equivalence. Suppose that $\lambda \in\left(\mathcal{L}_{n}\right)^{H} \cap\left(\mathcal{L}_{n}\right)_{\text {st }}^{J}$, and construct a new partition $\tilde{\lambda}$ by taking 
each $v \in \operatorname{cl}(\lambda)$, and refining it into its $J$-isotypic components. We claim that $\tilde{\lambda}$ is weakly fixed by $H$. Indeed, suppose the refinement $\tilde{\lambda}$ of $\lambda$ has $\operatorname{cl}(\tilde{\lambda})=\left\{v_{i}\right\}_{i \in \mathcal{I}}$ with $\oplus_{k} v_{i_{k}}=v_{i}$ and all of the $v_{i_{k}}$ are irreducible and isomorphic as representations of $J$. Let $h \in H$ and $j \in J$ be arbitrary, and let $x$ be an element of $v_{i_{k}}$. Then

$$
j h x=h h^{-1} j h x=h j^{\prime} x,
$$

for some $j^{\prime}$ in $J$. But $j^{\prime} x$ is an element of $v_{i_{k}}$. Thus we conclude that $h v_{i_{k}}$ is a representation of $J$.

Since $v_{i_{k}}$ is a $J$-representation, there is a corresponding map $\rho_{i_{k}}: J \rightarrow \operatorname{GL}\left(v_{i_{k}}\right)$ from $J$ to the linear automorphisms of $v_{i_{k}}$. Define $\rho_{i_{k}}^{h}: J \rightarrow \operatorname{GL}\left(v_{i_{k}}\right)$ by $\rho_{i_{k}}^{h}(j)=$ $\rho_{i_{k}}\left(h^{-1} j h\right)$. Since

$$
j h x=h\left(h^{-1} j h\right) x=h \rho_{i_{k}}^{h}(j) x,
$$

the map $x \mapsto h x$ defines an isomorphism from the representation determined by $\rho_{i_{k}}^{h}$ to $h v_{i_{k}}$. Since $\rho_{i_{k}}$ is irreducible, so is $\rho_{i_{k}}^{h}$. Thus $h v_{i_{k}}$ is irreducible. Moreover, if $v_{i_{k}}$ is isomorphic to $v_{i_{k^{\prime}}}$, then the representations corresponding to $\rho_{i_{k}}^{h}$ and $\rho_{i_{k^{\prime}}}^{h}$ are isomorphic as well, whence $h v_{i_{k}}$ is isomorphic to $h v_{i_{k^{\prime}}}$. It follows that $h v_{i}=\oplus_{k} h v_{i_{k}}$ is $J$-isotypic.

Since $h$ fixes $\lambda$, we have that $h$ permutes the elements of $\operatorname{cl}(\lambda)$. Let $w \in \operatorname{cl}(\lambda)$, and let $h w \in \operatorname{cl}(\lambda)$ denote its image. The previous paragraph shows that $h$ maps the $J$-isotypic components of $w$ to the $J$-isotypic components of $h w$. It follows that $H$ fixes the refinement of $\lambda$ into its $J$-isotypic components as claimed.

The original partition $\lambda$ is a coarsening of $\tilde{\lambda}$, so there is a natural transformation from the composite

$$
\left(\mathcal{L}_{n}\right)^{H} \cap\left(\mathcal{L}_{n}\right)_{\text {st }}^{J} \rightarrow\left(\mathcal{L}_{n}\right)^{H} \cap\left(\mathcal{L}_{n}\right)_{\text {iso }}^{J} \hookrightarrow\left(\mathcal{L}_{n}\right)^{H} \cap\left(\mathcal{L}_{n}\right)_{\text {st }}^{J}
$$

to the identity functor on $\left(\mathcal{L}_{n}\right)^{H} \cap\left(\mathcal{L}_{n}\right)_{\text {st }}^{J}$. If $\lambda$ is in $\left(\mathcal{L}_{n}\right)^{H} \cap\left(\mathcal{L}_{n}\right)_{\text {iso }}^{J}$, then $\tilde{\lambda}=\lambda$, so the map on classifying spaces induced by the functor $\lambda \mapsto \tilde{\lambda}$ is a deformation retraction, giving a homotopy equivalence

$$
\left(\mathcal{L}_{n}\right)^{H} \cap\left(\mathcal{L}_{n}\right)_{\text {st }}^{J} \simeq\left(\mathcal{L}_{n}\right)^{H} \cap\left(\mathcal{L}_{n}\right)_{\text {iso }}^{J} .
$$

The homotopy equivalences in equations (4.6) and (4.7), taken together, establish the weak equivalence required in the theorem.

It remains to show that $\left(\mathcal{L}_{n}\right)^{H} \cap\left(\mathcal{L}_{n}\right)_{\text {iso }}^{J}$ is contractible. Since we are assuming that $J$ is polytypic, the decomposition $\mu$ of $\mathbb{C}^{n}$ into the isotypic components of $J$ is a proper partition and is terminal in $\left(\mathcal{L}_{n}\right)_{\text {iso }}^{J}$. The partition $\mu$ is also in $\left(\mathcal{L}_{n}\right)^{H}$, by the same argument we used above to prove that the refinement of any $\lambda$ into its $J$-isotypic components was weakly fixed by $H$.

Thus $\mu$ is a terminal element in $\left(\mathcal{L}_{n}\right)^{H} \cap\left(\mathcal{L}_{n}\right)_{\text {iso }}^{J}$ showing that this category has contractible classifying space, as desired.

\section{Proof of the main theorem}

In this section, our goal is to use the results of the previous sections to prove Theorem 1.1. Let $H$ be a $p$-toral subgroup of $U(n)$ and let $\bar{H}$ be its image in $P U(n)$. Our plan is to pick out an appropriate element of order $p$ in $\bar{H}$, lift it to $U(n)$ using Lemma 3.1, and then use Theorem 4.5. 
Lemma 5.1. Let $H \subseteq U(n)$ be a subgroup with $\bar{H}$ its projection to $P U(n)$, and suppose there exists a subgroup $V \cong \mathbb{Z} / p$ of $\bar{H}$ such that

$$
V \subseteq \operatorname{ker}(\bar{H} \rightarrow \bar{H} / p)
$$

Then $V$ does not act transitively on $\operatorname{cl}(\lambda)$ for any weakly $H$-fixed partition $\lambda$ of $\mathbb{C}^{n}$.

Proof. Let $\lambda$ be an object of $\left(\mathcal{L}_{n}\right)^{H}$. Since $V \subseteq \bar{H}$, it follows that $V$ permutes the elements of $\operatorname{cl}(\lambda)$. We claim this permutation is not transitive. Assume the contrary. As $\lambda$ is a proper partition, it follows $|\operatorname{cl}(\lambda)|>1$, so $|\operatorname{cl}(\lambda)|=p$. We choose a bijection $\operatorname{cl}(\lambda) \cong\{1,2, \ldots, p\}$ such that the image of $V$ under the resulting map $\bar{H} \rightarrow \Sigma_{p}$ is generated by the $p$-cycle $(1,2, \ldots, p)$.

As $\bar{H}$ is $p$-toral, its image under the map $\bar{H} \rightarrow \Sigma_{p}$ is a $p$-group. By the assumption that $V$ permutes the elements of $\operatorname{cl}(\lambda)$ transitively, the image of $\bar{H}$ must contain the subgroup $\mathbb{Z} / p \subseteq \Sigma_{p}$ generated by $(1,2, \ldots, p)$. This subgroup is a maximal $p$-subgroup of $\Sigma_{p}$, which forces the image of $\bar{H}$ to be contained in $\mathbb{Z} / p$. Therefore $\bar{H} \rightarrow \Sigma_{p}$ factors through $\bar{H} \rightarrow \bar{H} / p$. Because the restriction to $V$ is nontrivial, the existence of this factorization contradicts the assumption that $V \subseteq \operatorname{ker}(\bar{H} \rightarrow \bar{H} / p)$. We conclude that $V$ cannot act transitively on $\operatorname{cl}(\lambda)$, thus proving the lemma.

Corollary 5.2. Let $H, \bar{H}$, and $V$ be as in Lemma 5.1, and suppose further that $V$ is normal in $\bar{H}$. Then $\left(\mathcal{L}_{n}\right)^{H}$ is contractible.

Proof. Let $J$ be the inverse image of $V$ in $H$; by Lemma 3.1, we know that $J$ is polytypic. By Lemma 5.1, $V$ does not act transitively on $\operatorname{cl}(\lambda)$ for any weakly $H$-fixed partition $\lambda$, and since the action of $J$ on $\mathcal{L}_{n}$ factors through $V$, we conclude that $J$ has the same property. Since $V$ is normal in $\bar{H}$, its inverse image $J$ is normal in $H$, so it follows that $J$ and $H$ satisfy the conditions of Theorem 4.5. Hence $\left(\mathcal{L}_{n}\right)^{H}$ is contractible.

We now have all the ingredients needed for the proof of our main theorem.

TheOREm 1.1. Suppose that $H$ is a p-toral subgroup of $U(n)$ and $\left(\mathcal{L}_{n}\right)^{H}$ is not contractible. Then $H$ is a projective elementary abelian p-subgroup of $U(n)$.

Proof. Let $H$ be a $p$-toral subgroup of $U(n)$. Assume that $\bar{H}$ is not a finite, elementary abelian $p$-group; then we want to prove that $\left(\mathcal{L}_{n}\right)^{H}$ is contractible. Since $\bar{H}$ is necessarily nontrivial, by Lemma 3.5 there exists

$$
V \cong \mathbb{Z} / p \subseteq Z(\bar{H}) \cap \operatorname{ker}(\bar{H} \rightarrow \bar{H} / p) .
$$

Thus we have $V \triangleleft \bar{H}$ that satisfies the hypotheses of Lemma 5.1 and Corollary 5.2 , and the theorem follows.

\section{Examples}

In this section we consider $p=2$ and compute two examples of fixed point sets $\left(\mathcal{L}_{n}\right)^{H}$ where $H$ is a projective elementary abelian $p$-group. We first consider an example $H \cong \mathbb{Z} / 2 \subseteq U(2)$ acting on $\mathcal{L}_{2}$, and the fixed points $\left(\mathcal{L}_{n}\right)^{H}$ turn out not to be contractible (Proposition 6.1). This example shows that Theorem 1.1 is group-theoretically sharp: there exist projective elementary abelian $p$-groups with noncontractible fixed point sets. The second example is $H^{\prime} \cong \mathbb{Z} / 2 \subseteq U(3)$ acting on $\mathcal{L}_{3}$. In this case the fixed point set turns out to be contractible (Proposition 6.3), 
illustrating that not all projective elementary abelian $p$-subgroups have noncontractible fixed point sets. In $\left[\mathbf{B J L}^{+}\right]$, we will establish further restrictions of a representation-theoretic nature that narrow down the $p$-toral subgroups of $U(n)$ that can have noncontractible fixed point sets on $\mathcal{L}_{n}$.

First we compute fixed points on $\mathcal{L}_{2}$. Let $H \cong \mathbb{Z} / 2 \subseteq U(2)$ be the subgroup generated by $\tau \in U(2)$ represented by the matrix $\left[\begin{array}{ll}0 & 1 \\ 1 & 0\end{array}\right]$.

Proposition 6.1. The fixed point space $\left(\mathcal{L}_{2}\right)^{H}$ is homeomorphic to the space $S^{1} \sqcup *$.

To prove Proposition 6.1, we set up a little notation. Let $L_{z}$ denote the line in $\mathbb{C}^{2}$ spanned by $(1, z)$, and let $L_{\infty}$ denote the line spanned by $(0,1)$. We saw in the proof of Proposition 2.1 that the set of objects in $\mathcal{L}_{2}$ consists of pairs $\left\{L_{z}, L_{-1 / \bar{z}}\right\}$ where $z \in \mathbb{C} \backslash\{0\}$, together with one extra point $\left\{L_{0}, L_{\infty}\right\}$. Since $\tau \in U(2)$ exchanges the standard basis vectors of $\mathbb{C}^{2}$, if $z \in \mathbb{C} \backslash\{0\}$ then $\tau\left(L_{z}\right)=L_{1 / z}$, and $\tau$ exchanges $L_{0}$ and $L_{\infty}$.

Lemma 6.2. As a set, the fixed points of the action of $\tau$ on $\mathcal{L}_{2}$ consist of the point $\left\{L_{1}, L_{-1}\right\}$, the point $\left\{L_{0}, L_{\infty}\right\}$, and the set of points $\left\{L_{i r}, L_{-i / r}\right\}$ where $r \in \mathbb{R} \backslash\{0\}$.

Proof. Direct computation establishes that the points of $\mathcal{L}_{2}$ in the statement of the lemma are in fact fixed by $\tau$.

Points in $\mathcal{L}_{2}$ besides $\left\{L_{0}, L_{\infty}\right\}$ have the form $\left\{L_{z}, L_{-1 / \bar{z}}\right\}$ where $z \in \mathbb{C} \backslash\{0\}$. If such a point is fixed by $\tau$, then either each line in the pair is fixed by $\tau$ (the partition is strongly fixed), or else the lines are interchanged by $\tau$ (the partition is only weakly fixed). In the first case, since $\tau\left(L_{z}\right)=L_{1 / z}$, we must have $z=1 / z$, so $z= \pm 1$, corresponding to the point $\left\{L_{1}, L_{-1}\right\}$. In the second case, we must have $1 / z=-1 / \bar{z}$, meaning $\bar{z}=-z$, so $z$ is purely imaginary, say $z=i r$ for $r \in \mathbb{R} \backslash\{0\}$. Thus $\left\{L_{z}, L_{-1 / \bar{z}}\right\}$ has the form $\left\{L_{i r}, L_{-i / r}\right\}$.

Proof of Proposition 6.1. To determine the fixed point set of the action of $\tau$ on $\mathcal{L}_{2}$ as a topological space, and not just as a set (as in Lemma 6.2), we recall from the proof of Proposition 2.1 that $\mathcal{L}_{2}$ can be identified as the quotient space of the disk $\|z\| \leq 1$ in $\mathbb{C}^{1}$ by the antipodal action on the boundary circle $\|z\|=1$. According to Lemma 6.2, the fixed points of $\tau$ correspond to the points of the unit disk that lie on the imaginary axis, $\{$ ir $\mid r \in[-1,1] \subseteq \mathbb{R}\}$, together with the real points 1 and -1 . The points 1 and -1 are identified by passing to $\mathcal{L}_{2}$, as are the points $i$ and $-i$, which gives $S^{1} \sqcup *$ as the fixed point space of $\tau$ acting on $\mathcal{L}_{2}$.

Proposition 6.1 gives an example of a $\mathbb{Z} / 2 \subseteq U(2)$ that acts with noncontractible fixed point set on $\mathcal{L}_{2}$. However, when we take the same subgroup and embed it in $U(3)$, we get a different result. Let $H^{\prime} \cong \mathbb{Z} / 2 \subseteq U(3)$ be the subgroup generated by

$$
\tau^{\prime}=\left[\begin{array}{lll}
0 & 1 & 0 \\
1 & 0 & 0 \\
0 & 0 & 1
\end{array}\right] \in U(3)
$$


Proposition 6.3. The fixed point space $\left(\mathcal{L}_{3}\right)^{H^{\prime}}$ is contractible.

Proof. We use essentially the proof of Theorem 4.5, slightly modified.

First, we assert that if $\lambda$ is weakly fixed by $H^{\prime}$, then the partition $\left(\lambda / H^{\prime}\right)$ described in Definition 4.3 is a proper partition of $\mathbb{C}^{3}$. There are two cases: either $\operatorname{cl}(\lambda)$ consists of three lines, in which case $H^{\prime} \cong \mathbb{Z} / 2$ cannot act on it transitively, or else $\lambda$ has one line and one two-dimensional subspace, in which case $H^{\prime}$ cannot interchange them because they have different dimensions. As a result, the inclusion

$$
\left(\mathcal{L}_{3}\right)_{\mathrm{st}}^{H^{\prime}} \hookrightarrow\left(\mathcal{L}_{3}\right)^{H^{\prime}}
$$

has a functorial retraction $\lambda \mapsto\left(\lambda / H^{\prime}\right)$, and induces a homotopy equivalence.

Exactly as in the proof of Theorem 4.5, we note that

$$
\left(\mathcal{L}_{3}\right)_{\text {iso }}^{H^{\prime}} \hookrightarrow\left(\mathcal{L}_{3}\right)_{\text {st }}^{H^{\prime}}
$$

also has the functorial retraction that takes an object $\lambda$ in $\left(\mathcal{L}_{3}\right)_{\text {st }}^{H^{\prime}}$ to its refinement into $H^{\prime}$-isotypic classes. Therefore each of the inclusions

$$
\left(\mathcal{L}_{3}\right)_{\text {iso }}^{H^{\prime}} \hookrightarrow\left(\mathcal{L}_{3}\right)_{\text {st }}^{H^{\prime}} \hookrightarrow\left(\mathcal{L}_{3}\right)^{H^{\prime}}
$$

induces a homotopy equivalence.

To finish the proof we need to identify the homotopy type of $\left(\mathcal{L}_{3}\right)_{\text {iso }}^{H^{\prime}}$. The action of $\tau^{\prime}$ on $\mathbb{C}^{3}$ has eigenvalues 1 (with multiplicity 2) and -1 . Therefore $H^{\prime}$ acts polytypically on $\mathbb{C}^{3}$, and $\left(\mathcal{L}_{3}\right)_{\text {iso }}^{H^{\prime}}$ has a final object consisting of the canonical decomposition of $\mathbb{C}^{3}$ into isotypic representations of $H^{\prime}$ :

$$
\mu=\{\{(u, u, v) \mid u, v \in \mathbb{C}\},\{(u,-u, 0) \mid u \in \mathbb{C}\}\}
$$

We conclude that $\left(\mathcal{L}_{3}\right)^{H^{\prime}} \simeq\left(\mathcal{L}_{3}\right)_{\text {st }}^{H^{\prime}} \simeq\left(\mathcal{L}_{3}\right)_{\text {iso }}^{H^{\prime}} \simeq *$.

\section{References}

[ADL13] Gregory Z. Arone, William G. Dwyer, and Kathryn Lesh, Bredon homology of partition complexes, arXiv preprint arXiv:1306.0056 [math.AT], 2013.

[AL07] Gregory Z. Arone and Kathryn Lesh, Filtered spectra arising from permutative categories, J. Reine Angew. Math. 604 (2007), 73-136. MR 2320314 (2008c:55013)

[Aro02] Gregory Z. Arone, The Weiss derivatives of $B \mathrm{O}(-)$ and $B \mathrm{U}(-)$, Topology 41 (2002), no. 3, 451-481. MR 1910037 (2003c:55012)

$\left[\mathrm{BJL}^{+}\right]$Julia E. Bergner, Ruth Joachimi, Kathryn Lesh, Vesna Stojanoska, and Kirsten Wickelgren, Classification of problematic p-toral subgroups of $U(n)$, in preparation.

[GW98] Roe Goodman and Nolan R. Wallach, Representations and invariants of the classical groups, Encyclopedia of Mathematics and its Applications, vol. 68, Cambridge University Press, Cambridge, 1998. MR 1606831 (99b:20073)

[Lib11] Assaf Libman, Orbit spaces, Quillen's theorem A and Minami's formula for compact Lie groups, Fund. Math. 213 (2011), no. 2, 115-167. MR 2800583 (2012f:55010)

[MT91] Mamoru Mimura and Hirosi Toda, Topology of Lie groups. I, II, Translations of Mathematical Monographs, vol. 91, American Mathematical Society, Providence, RI, 1991, Translated from the 1978 Japanese edition by the authors. MR 1122592 (92h:55001) 
Department of Mathematics, University of California, Riverside

E-mail address: bergnerj@member.ams.org

Department of Mathematics and Informatics, University of Wuppertal, Germany

E-mail address: joachimi@math.uni-wuppertal.de

Department of Mathematics, Union College, Schenectady, NY 12309 USA

E-mail address: leshk@union.edu

Department of Mathematics, Massachusetts Institute of Technology, Cambridge MA 02143

E-mail address: vstojanoska@math.mit.edu

Georgia Institute of Technology, School of Mathematics, 686 Cherry Street, AtLANTA GA 30332

E-mail address: wickelgren@post.harvard.edu 